\title{
Role of sonohysterography in evaluation of abnormally thickened endometrium causing abnormal uterine bleeding with histopathological correlation
}

\author{
Parveen $\mathrm{S}^{1}$, Azad SA ${ }^{2}$, Mohammad J ${ }^{1}$, Haque $\mathrm{S}^{3}$, Shanta $\mathrm{RS}^{1}$, Rahman $\mathrm{SM}^{2}$ \\ ${ }^{I}$ Department of Radiology and Imaging, United Hospital, Dhaka, ${ }^{2}$ Department of Radiology and \\ Imaging, Bangabandhu Sheikh Mujib Medical University, Shahbag, Dhaka, \\ ${ }^{3}$ Department of Radiology and Imaging, Dhaka Medical College \& Hospital, Dhaka. \\ Email: shakila_31@yahoo.com
}

\begin{abstract}
This prospective randomized study was carried out in the department of Radiology and Imaging in collaboration with the department of Obstetrics and Gynecology of Bangabandhu Sheikh Mujib Medical University (BSMMU), Shahbag and Dhaka Medical College Hospital (DMCH) during the period of January 2003 to December 2004. The aim of this study was to establish sonohyterography as a sensitive tool and superior to Transvaginal Sonography (TVS) for detection of endometrial pathology in women with abnormal uterine bleeding (AUB) and its validity by determining sensitivity, specificity, accuracy, positive predictive value and negative predictive value. For this purpose, a total number of 40 patients who had thickened endometrium (cut off level $<15 \mathrm{~mm}$ for pre menopausal and $<5 \mathrm{~mm}$ for post menopausal patients) detected on transabdominal or transvaginal sonography were selected. TVS and TV-SH were done in all these patients and findings were then compared with the final histopathological diagnosis. In our study, we found TV-SH has sensitivity of $100 \%$, specificity of $80 \%$ and accuracy of $97.5 \%$ compared to TVS (94\%, $66.66 \%$ and $90 \%$ respectively) in detection of endometrial pathology in women with AUB. TV-SH can thus be regarded as one of the most sensitive, specific and accurate modality in detecting and excluding endoluminal masses as the cause of AUB. It can be considered as the initial examination in the evaluation of all women with AUB.
\end{abstract}

\section{Introduction}

Abnormal uterine bleeding (AUB) is a common reason for gynecological visits of both pre menopausal and postmenopausal women ${ }^{1}$. Up to $33 \%$ of women referred to gynecological out patient department have AUB and this proportion rises to $69 \%$ in peri and post menopausal group ${ }^{2}$. AUB may be due to organic causes or dysfunctional. Organic causes may be systemic like underlying bleeding disorder or local such as endometrial polyps, hyperplasia, carcinoma and fibroids. Endometrial pathology associated with AUB often cause thickening of endometrium which might be diffuse or focal. Endometrial thickening is a non specific finding which is seen with secretory endometrium, estrogen therapy, early pregnancy, endometrial hyperplasia, polyps and endometrial carcinoma. Sub endometrial pathologic condition such as submucosal myoma and adenomyosis may also manifest as apparent endometrial thickening ${ }^{3}$. Abdominal or transvaginal sonography can identify thickened endometrium but unable to differentiate between potential etiologies of thickened endometrium like polyps, endometrial hyperplasia, submucous fibroid or endometrial carcinoma.

With instillation of fluid into endometrial canal, sonohystergraphy allows differentiation between focal and diffuse endometrial or subendometrial pathologic condition, which often leads to a specific diagnosis. Thus TV-SH can improve the specificity of TVS in differentiating endoluminal masses from diffuse endometrial thickening ${ }^{4}$. MRI is an important technique in the evaluation of uterine pathology due to its ability to obtain images with high soft tissue contrast resolution, multiplanner imaging facility and lack of ionizing radiation ${ }^{5}$. But in our country this modality is expensive and less available. So, Sonohysterography is an easy, safe, rapid and tolerable procedure which has an excellent diagnostic accuracy in differentiation of different endometrial pathology. On the basis of above facts, this study was designed to establish Sonohysterography as a sensitive tool and superior to TVS used alone for evaluation of focal and diffuse endometrial 
pathology. The aim and objective of this study is to find out the value of Sonohysterography in the diagnosis and management of AUB and to elucidate sensitivity, specificity and accuracy of Sonohysterography in evaluation of abnormally thickened endometrium in women with AUB with histopathological correlation.

\section{Materials and Methods}

This prospective study was carried out in the department of Radiology and Imaging in collaboration with the department of Obstetrics and Gynecology of BSSMMU and DMCH during the period of January 2003 to December 2004. This study was done on 40 patients ranging from 32 to55 years of age range with abnormal uterine bleeding (menorrhagia, metrorrhagia, menometrorrhagia and post menopausal bleeding) and referred for USG of pelvic organs. Only the patients having thickened endometrium on ultrasonography $>5 \mathrm{~mm}$ in postmenopausal women and $>15 \mathrm{~mm}$ in pre menopausal women with AUB were included. Patients having AUB due to causes other than endometrial pathology, those who were unfit or unwilling to do TVS and those with unavailability of histopathological reports were excluded.

At first all patients were evaluated on the basis of detailed history, clinical assessment, relevant laboratory investigations and subsequently they underwent trans abdominal sonography. After giving a brief description about TVS and TV-SH , both the procedures were done only in those patients having thickened endometrium.

TVS was carried out with $5 \mathrm{MHz}$ and $7.5 \mathrm{MHz}$ multi-frequency convex transvaginal probe. Saggital and coronal TVS images of uterus were obtained in all patients. Double layer thickness was determined in mid saggital view. The only preparation required was for the patient to empty the bladder. TV-SH was carried out immediately after the performance of TVS. It was performed by placing the patient in lithotomy position and speculum was placed into vagina to expose the cervix. The external os was cleansed with povidone-iodine (betadine). The anterior lip of cervix was gripped by a volsellum and a 5-F catheter was advanced into the cervical canal. The speculum was then removed and the sterile sheathed transvaginal probe was reintroduced and approximately 5-10 $\mathrm{ml}$ of sterile saline was injected slowly through the catheter under direct sonographic visualization. Scanning was maintained during saline infusion and selected pictures were taken in different planes. Endometrial echo, regularity and shape were depicted and endometrial thickness surrounding the fluid was measured on mid saggital view. The findings of TVS and TV-SH were correlated with histopathological examination. The specimens were obtained by endometrial curettage and /or hysterectomy specimen.

For the validity of the study outcome, sensitivity, specificity, accuracy, positive and negative predictive value of TVS and TV-SH in the diagnosis of endometrial pathology were calculated after confirmation of the diagnosis by histopathology and were calculated by standard formula. Statistical tests for significance of differences were done using ' $Z$ ' test where applicable like thickness of different endometrial pathology, distribution of endometrial pathologies in different age groups. A p-value <.05 was considered as significant.

\section{Results}

The study included 40 women whose age ranged from 32 to 55 years with abnormal uterine bleeding. They were divided into $30-40$ years, 4050 years and 50-60 years age groups and their numbers were $16(40 \%), 18(45 \%)$ and $6(15 \%)$ in each age group respectively. The mean age was 39 years. Highest incidence was found in perimenopausal women (40-50 years) among which endometrial hyperplasia was the commonest. The highest thickness of endometrium was found in endometrial polyp.

Most common symptom associated with endometrial pathology was abnormal per vaginal bleeding in all 40 cases (100\%) followed by 18 cases $(45 \%)$ with lower abdominal pain, 10 cases (25\%) with infertility/sub fertility and 5 cases (12.55) with dysmenorrhea.

Among 40 patients TVS diagnosed 17 cases (40\%) of endometrial hyperplasia, most of which appeared as diffuse and homogenous thickening of echogenic endometrium. Thickness was $>16 \mathrm{~mm}$ in pre and $>8 \mathrm{~mm}$ in postmenopausal patients while other showed focal and cystic hyperplasia. 11 cases of endometrial polyps were detected by TVS which appeared as well defined focal thickening of endometrium of $2-4 \mathrm{~cm}$ with preservation of endometrium- myometrium interface. TVS failed to detect both two cases of endometrial carcinoma, one case was misdiagnosed as endometrial hyperplasia and the other as endometrial polyp. Out of 8 cases 6 were correctly diagnosed as subendometrial fibroids which appeared as hyper or mixed echogenic solid mass with an overlying 
endometrium pushed and distorted by the mass. 4 true negative cases were endoluminal blood clot.

TV-SH diagnosed all the cases of endometrial hyperplasia and subendometrial fibroid. Endometrial hyperplasia appeared as diffuse or focal thickening of endometrium well visualized in presence of intracavitory fluid. Endometrial polyps were typically well defined, polypoid, homogenous mass usually isoechoic to endometrium [fig. 2(a), 2(b)]. One case of endometrial carcinoma was missed by TV-SH and considered as polyp. Submucosal fibroids were well defined, broad based hyper/ hypo/ heterogenous mass which distorted the myometrium-endometrium interface. 4 true negative cases were intraluminal blood clot.

17 cases were detected as endometrial hyperplasia by TVS. Out of these 16 cases were correctly diagnosed and the rest was endometrial carcinoma histologically. Out of 11 cases of endometrial polyp detected by TVS, 10 cases were histopathologically diagnosed. It failed to detect all the two cases of endometrial carcinoma and two cases of subendometrial fibroids. Among 40 patients, 32 patients were true positive and 2 were false positive. 4 patients had apparently thickened endometrium due to presence of intraluminal clot or retained product of conception and considered as true negative. 2 cases were false negative, as TVS failed to detect 2 cases of subendometrial fibroid.

TV-SH detected 16 cases of endometrial hyperplasia and 8 cases of subendometrial fibroids which were also proved histopathologically. Out of two cases of endometrial carcinoma one was missed by TV-SH and considered as endometrial polyp. Among 40 patients, 35 patients were true positive and 1 was false positive. 4 patients had apparently thickened endometrium due to presence of intraluminal clot or retained product of conception and considered as true negative. No false negative cases were detected.

In our study, we found TV-SH has sensitivity of $100 \%$, Specificity of $80 \%$ and accuracy of $97.5 \%$ compared to TVS $(94 \%, 66.66 \%$ and $90 \%$ respectively) in detection of endometrial pathology in women with AUB (Table I). Our study result as compared to histopathology is reflected in fig. 1 .

Table I: Showing the validity of TVS and TV-SH findings with histopathological diagnosis

\begin{tabular}{lcc}
\hline Diagnostic accuracy & $\begin{array}{c}\text { TVS } \\
\text { (Percentage) }\end{array}$ & $\begin{array}{c}\text { TV-SH } \\
\text { (Percentage) }\end{array}$ \\
\hline Sensitivity & 94.11 & 100 \\
Specificity & 66.66 & 80 \\
Accuracy & 90 & 97.5 \\
Positive predictive value & 94.11 & 97.22 \\
Negative predictive value & 66.66 & 100 \\
\hline
\end{tabular}

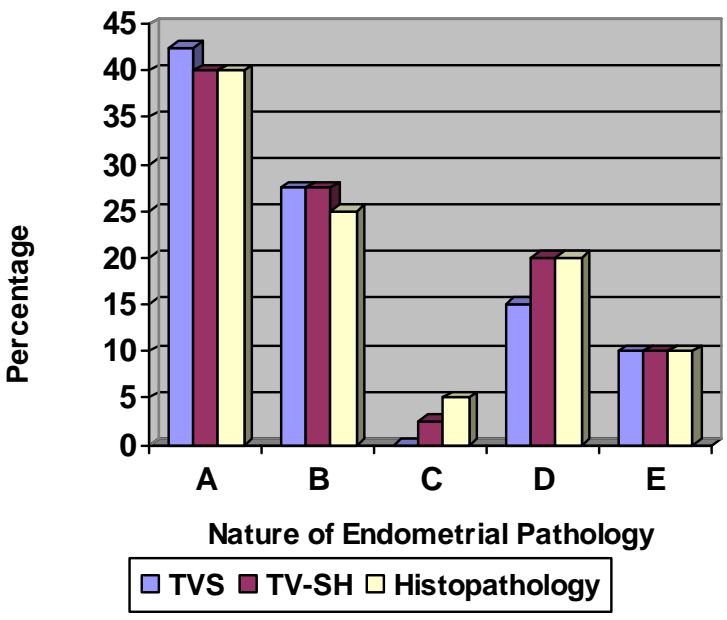

A- Endometrial Hyperplasia, B-Endometrial Polyp C- Endometrial Carcinoma, D-Sub endometrial fibroid E- Endo luminal blood clot

Fig. 1: Bar diagram showing endometrindal abnormality detected by TVS and TV-SH with histopathological correlation

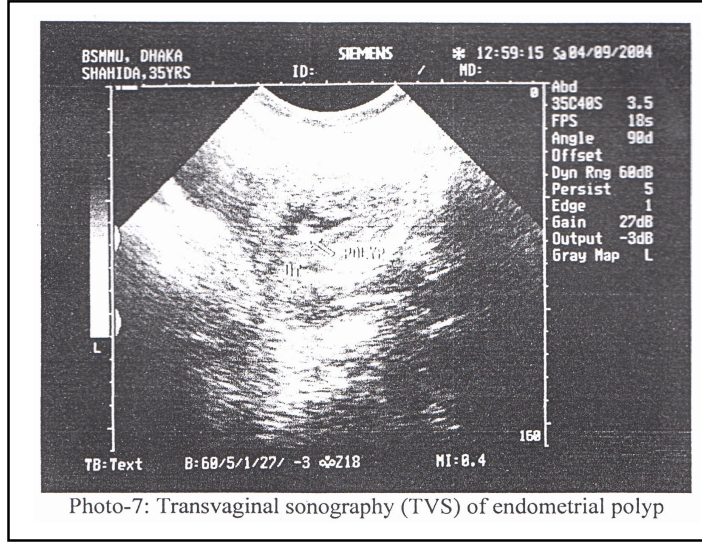

Fig. 2 (a): Endometrial polyp as shown in TVS.

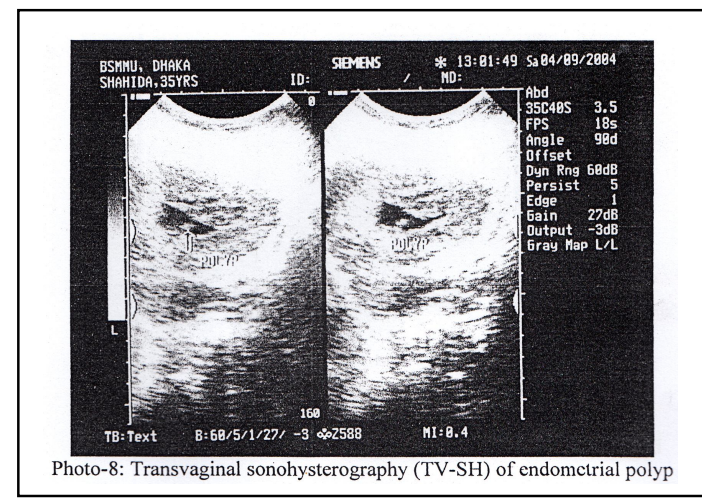

Fig. 2 (b): Endometrial polyp as shown in TV-SH.

\section{Discussion}

During the last two decades tremendous advancement has occurred in the technology of diagnostic imaging and ultrasonography arena is also in front step with the development of newer generation equipment and expertise. In gynecology, 
TVS is used increasingly as it is a safe, available and sensitive modality for non-invasive imaging of endometrial cavity due to proximity of the endovaginal probe to the endometrium. Sensitivity of TVS can be increased by instillation of fluid into uterine cavity (TV-SH).

Out of 40 patients there were 16 cases of endometrial hyperplasia, 10 cases of endometrial polyp, 2 cases of endometrial carcinoma, 8 subendometrial fibroids and 4 cases had intra luminal blood clot which were the true negative cases. The most common endometrial pathology was endometrial hyperplasia common in 40-50 years age group and the mean age was 42.8 years. Similar results were obtained by Tabbakh and Slamaka, 2002 where the mean age was 44.34 years ${ }^{6}$.

In our study, we found TV-SH has 100\% sensitivity, specificity and accuracy compared to TVS $(100 \%, 96 \%$ and $97.5 \%)$ in detection of endometrial hyperplasia. The result is almost close to the study of Tabbakh and Slamaka, 2002 who found that TV-SH has sensitivity of $95 \%$, specificity $96.8 \%$ and diagnostic accuracy $96.5 \%$ compared to TVS $(77 \%, 94.6 \%$ and $89.8 \%$ ) for endometrial hyperplasia.

In this study $33 \%$ women with postmenopausal bleeding was due to endometrial carcinoma and both the cases were over 50 years of age. This agrees totally with Ross, $1998^{7}$. The sensitivity, specificity of TV-SH in detection of endometrial carcinoma in current study was 50\% and 100\% respectively which are significantly different compared to the study carried out by Dubinsky et al. 1997, where sensitivity was $89 \%$ and specificity $46 \%$. This difference can be honestly attributed to more experience which is an important factor.

In a study of 72 cases by Becker et al. 2002, sensitivity value for submucous myoma and focal endometrial lesion were $100 \%$ and $90 \%$ respectively for TV-SH and 100\% and $70 \%$ respectively for TVS alone?. This study showed sensitivity value for submucous myoma is $75 \%$ and for endometrial polyp is $100 \%$ by TVS alone. Whereas TV-SH showed $100 \%$ sensitivity for both submucous myoma and endometrial polyp, these findings are close to the study of Becker et al.

In another study by Cacciatore et al, 1989 the sensitivity and specificity of TVS in detection of endometrial pathology were $73.9 \%$ and $95.7 \%$ respectively ${ }^{10}$. In current study, the sensitivity, specificity and accuracy of TVS in analysis of endometrial pathology is $94 \%, 66.66 \%$ and $90 \%$ respectively.
In a study done in 1995, Dubinsky et al.found that TV-SH can improve the specificity of TVS in differentiating endoluminal masses from more diffuse endometrial thickening ${ }^{11}$.

On the other hand, in this present series the sensitivity of TV-SH was $100 \%$, specificity $80 \%$ and diagnostic accuracy $97 \%$ in differentiation of endometrial pathology. TV-SH provided information over TVS which is similar to the study of Becker et al., 2002 who concluded that TV-SH is an important adjunct to TVS ${ }^{9}$.

So, TV-SH is more sensitive, specific and accurate in diagnosis of focal and diffuse endometrial and sub endometrial lesions. TV-SH can improve the specificity of TVS in differentiating endoluminal masses from more diffuse endometrial thickening because it allows reliable differentiation between focal and diffuse endometrial and sub endometrial lesions (Davis et al., 2002) ${ }^{\mathbf{1 2}}$.

Following our study, several research works were also done to detect the effectiveness of TV-SH in assessment of different endometrial pathology resulting AUB and their results closely match with those of ours.

Azab et al., 2006 concluded that Sonohysterography has further enhanced the transvaginal ultrasound examination of the uterine cavity. This can be used for further evaluation of the site, nature, and even reach a final diagnosis of the lesion yet it could not replace the histopathologic diagnosis, however it can help determine the best mode of the treatment, whether medical or surgical ${ }^{13}$.

The study result of Aslam et al., 2007 substantiated that sonohysterography is a better tool than transvaginal sonography for the assessment of endometrial intra-cavity lesions and recommend that saline contrast sonohysterography should be used as an initial investigation in cases of abnormal uterine bleeding ${ }^{14}$.

In a study by Erdem et al., in 2007 the sensitivity and specificity of TV-SH were $97.7 \%$ and $82.4 \%$, respectively versus $83.0 \%$ and $70.6 \%$, respectively for TVUS for detection of endometrial abnormalities such as polyps, submucosal fibroids, and endometrial hyperplasia in patients with abnormal vaginal bleeding ${ }^{\mathbf{1 5}}$. This test validity is almost similar to our study result.

In summary of literature review, Bennett et al.in 2010 stated that TV-SH helps better delineate the endometrium when it cannot be well-visualized at TVS $^{16}$. 
In a study done in 2011, Wolfman and his colleagues concluded that TV-SH and MRI are helpful in equivocal cases or when TVS is nondiagnostic. As MRI is less available in our country and also expensive, saline contrast sonohysterography can be used reliably as an initial investigation in cases of abnormal uterine bleeding $^{17}$.

Conclusion: As the TV-SH finding of the present study correlated well with the histopathology findings and the validity test values were higher than that with TVS which were almost identical or even higher than observed by others it can be concluded that TV-SH is sensitive and accurate modality in the evaluation of causes of thickened endometrium. It can be considered as the initial examination in the evaluation of all women with AUB.

\section{References}

1. Karampl E, Bourne T, Harlen-Solbakken H, Istre O. Transvaginal sonography, sonohysterography and operative hysteroscopy for the evaluation of AUB. Acta. Obstet, Gynaecol Scand 2001; 80(7): 616-622.

2. Mencaglia L, Perino A, Hamou J, Hysteroscopy in perimenopausal and post menopausal women with AUB. J Reprod Med 1987; 32: 577-582.

3. Levine D, Gosink BB, Johnson LA. Change in endometrial thickness in women undergoing hormone replacement therapy. Radiology 1995; 197: 603-608.

4. Jorizzo JR, Riccio GJ, Chen MYM and Carr JJ. Sonohysterography: The next step in the evaluation of the abnormal endometrium. Radiographics, 1999; 19 (90001): 117-130.

5. Lees WR, Highman JH (1998) Gynecological imaging. Sutton D (editor) Text book of Radiology and Imaging, $6^{\text {th }}$ edition, London: Churchill Livingstone, pp. 12351250 .

6. Tabbakh MNE, Slamaka P. Correlation between transvaginal sonography, Sonohysterography, Hysterosalphingography and operative hysteroscopy in predicting endometrial hyperplasia. J. Egyp Soc obstet and gynecol 2002; 18(3): 21-32.
7. Ross L. Pelvic ultrasound- should it be used routinely in the diagnosis and treatment of post menopausal bleeding? J. Royal Soc. Med 1998; 81: 723-724.

8. Dubinsky JT, Parvey RH, Maklad N. The role of transvaginal sonography and endometrial biopsy in the evaluation of peri and postmenopausal bleeding. AJR 1997; 169: 145-149.

9. Becker E, Lev- Toaff AS, Kaufman EP, Halpern EJ, Edelweiss et al. The added value of transvaginal sonohysterography over TVS alone in women with known or suspected leiomyoma. J. Ultrasound med. 2002; 21(3): 237- 247.

10. Cacciatore B, Lehtovitra P, Wahlstrom T, Ylstalo P.Preoperative sonographic evaluation of endometrial cancer. Am j Obstet Gynecol 1989; 160: 133-137.

11. Dubinsky JT, Parvey RH, Gormaz G, Maklad N Transvaginal sonohysterography in evaluation of small endometrial mass. J Ultrasound Med 1995; 14: 1-6.

12. Davis PC, O'Neil MJ, Yoder IC, Lee SI, Mueller PR. Sonohysterographic finding of endometrial and subendometrial conditions. Radiographics 2002; 22(4): 803-816.

13. Azab HI, El Nowiem KI, El Ghitany MA, El Din Mohamed FS. Role of sonohysterography in evaluation of abnormal uterine bleeding. Bull. Alex. Fac. Med 2006; 42(3): 668-669.

14. Aslam M, Ijaz L, Tariq S, Shafqat K, Meher-un-Nisa, Ashraf $R$ et al. Comparison of Transvaginal Sonography and Saline Contrast onohysterography in Women with Abnormal Uterine Bleeding: Correlation with Hysteroscopy and Histopathology. Int J Health Sci (Qassim). 2007; (1): 17-24.

15. Erdem M, Bilgin U, Bozkurt N, Erdem A. Comparison of transvaginal ultrasonography and saline infusion sonohysterography in evaluating the endometrial cavity in pre and postmenopausal women with abnormal uterine bleeding. Menopause 2007; 14(5): 846-852.

16. Genevieve L. Bennett, Rochelle F. Andreotti, Susanna I. Lee, Sandra O. DeJesus Allison, Douglas L. Brown, Theodore Dubinsky et al. ACR Appropriateness Criteria $^{\circledR}$ on Abnormal Vaginal Bleeding. Review article, Journal of the American College of Radiology 2011; 8(7): 460-468.

17. Darcy J. Wolfman, Sandra J. Allison and Susan M. Ascher. Imaging of benign uterine conditions. Applied Radiology Online Journal, 2011; 40: 11. 